\title{
Energy loss of ions at metal surfaces: Band-structure effects
}

\author{
M. Alducin \\ Departamento de Ingeniería Eléctrica, ETSII, UPV/EHU, Alda. de Urquijo s/n, E-48013 Bilbao, Spain \\ V. M. Silkin \\ Donostia International Physics Center (DIPC), P. Manuel de Lardizabal 4, 20018 San Sebastián, Spain
}

\begin{abstract}
J. I. Juaristi and E. V. Chulkov
Departamento de Física de Materiales and Centro Mixto CSIC-UPV/EHU, Facultad de Químicas, UPV/EHU, Apartado 1072, 20080 San Sebastián, Spain
\end{abstract}

(Received 6 November 2002; published 25 March 2003)

\begin{abstract}
We study band-structure effects on the energy loss of protons scattered off the $\mathrm{Cu}$ (111) surface. The distance dependent stopping power for a projectile traveling parallel to the surface is calculated within the linear response theory. The self-consistent electronic response of the system is evaluated within the random-phase approximation. In order to characterize the surface band structure, the electronic single-particle wave functions and energies are obtained by solving the Schrödinger equation with a realistic one-dimensional model potential. This potential reproduces the main features of the $\mathrm{Cu}(111)$ surface: the energy band gap for electron motion along the surface normal, as well as the binding energy of the occupied surface state and the first image state. Comparison of our results with those obtained within the jellium model allows us to characterize the band-structure effects in the energy loss of protons interacting with the $\mathrm{Cu}$ (111) surface.
\end{abstract}

DOI: 10.1103/PhysRevA.67.032903

PACS number(s): 34.50.Bw, 73.20.At

\section{INTRODUCTION}

The study of the energy loss of ions interacting with metal surfaces has constituted an active field of research during the last years [1]. Many experiments have provided information about the energy losses experienced by proton [2-4] and heavy ion projectiles [5-7] under grazing scattering conditions.

In the analysis of these experiments a central quantity is the so-called distance dependent stopping power, i.e., the energy lost per unit path length traveled by the ion parallel to the metal surface, as a function of the ion-surface separation. Echenique and Pendry [8] used a local response function to calculate this quantity and applied it to the calculation of the energy losses of fast electrons traveling parallel to the surface. In latter works, wave-vector-dependent surface dielectric functions were used [9-14]. These calculations were based on the specular-reflection model (SRM) $[15,16]$. This model allows one to obtain the surface response function in terms of the bulk response function. The approximation used in the SRM assumes that the conduction electrons are confined by an infinite potential barrier at the surface, and the quantum interference between the outgoing and ingoing components of the electrons reflected at this barrier is neglected. In a different approach Gravielle and collaborators $[17,18]$ have studied the interaction of a charged particle with the conduction electrons bounded by a finite step potential at the surface. This model describes single-particle excitations (electron-hole creation), but does not include collective excitations (plasmons). In the up-to-now most advanced calculations of the distance-dependent stopping power [1921], the Kohn-Sham formulation [22] of the density functional theory [23] was used to calculate self-consistently a finite and smooth surface potential barrier. In these works, the jellium model was used to describe the conduction band of the metal: the ionic background made up of nuclei and core electrons was replaced by a uniform positive charge distribution. In Refs. $[19,20]$ the Kohn-Sham orbitals were used to construct the response function of the system in the random-phase approximation (RPA) [24,25]. In Ref. [21] exchange and correlation effects were also included in the calculation of the response within the so-called adiabatic local density approximation [26]. Nevertheless, it was observed that the stopping power for a particle traveling parallel to a metal surface was almost insensitive to this correction. On the contrary, the use of a self-consistenly calculated finite surface barrier improved significantly the results obtained within the SRM, for both the stopping power and the total energy loss, up to velocities of the order of $v=2 v_{0}$ to $3 v_{0}$ $\left(v_{0}\right.$ is the Bohr velocity) $[19,20]$. At higher velocities, the SRM showed a reliable approximation.

All these works invoke the jellium model. In a real solid, the valence electrons move in a periodic potential, and the electronic states are described by the Bloch wave functions. In this work we aim to study how this affects the energy loss of ions under grazing incidence. For this purpose, we incorporate information about the band structure of a solid in the calculation of the distance-dependent stopping power for an ion traveling parallel to the $\mathrm{Cu}$ (111) surface.

The $\mathrm{Cu}$ (111) surface is a good candidate for our study because it permits us to analyze the effect of two important characteristics that its band structure presents: (1) the gap exhibited by the bulk band structure for the electron motion along the surface normal between $-5.83 \mathrm{eV}$ and $0.69 \mathrm{eV}$, and (2) the occupied surface state located at $-5.33 \mathrm{eV}$ (see Ref. [27], and references therein). All these energies are measured from the vacuum level.

The outline of the paper is the following: in Sec. II the 
model used is explained, in Sec. III the results obtained are presented and are compared to those obtained within the jellium model, and in Sec. IV we summarize the main conclusions of this work.

Atomic units (a.u.) will be used unless it is otherwise stated.

\section{MODEL}

When a swift charged particle interacts with matter, it induces a polarization potential $V^{\text {ind }}(\mathbf{r}, t)$. This potential acts back on the projectile giving rise to a retarding force. The energy that the particle loses per unit time due to this force can be calculated in the following way [21]:

$$
\frac{d E}{d t}=-\int d \mathbf{r} \rho^{e x t}(\mathbf{r}, t) \frac{\partial V^{i n d}(\mathbf{r}, t)}{\partial t},
$$

where $\rho^{\text {ext }}(\mathbf{r}, t)$ represents the incident-particle charge density.

Henceforth, we take $z$ as the coordinate of the position vector normal to the surface. The topmost atomic layer is at $z=0$ and the solid is in the $z<0$ side. We use capital letters for the coordinates parallel to the surface $[\mathbf{r}=(\mathbf{R}, z)]$. Here, we study the case of a point charge $Z_{1}$ moving parallel to the surface with velocity $\mathbf{v}$ at distance $z_{0}>0$ :

$$
\rho^{e x t}(\mathbf{r}, t)=Z_{1} \delta(\mathbf{R}-\mathbf{v} t) \delta\left(z-z_{0}\right) .
$$

We assume translational invariance in the plane parallel to the surface, i.e., we neglect surface corrugation effects. This is a reasonable approximation considering that we restrict ourselves to the study of randomly incident ions. In this case, within linear response theory, the potential induced by an external charge can be calculated in terms of the twodimensional Fourier transform of the induced part of the screened interaction $W^{\text {ind }}\left(Q, z, z^{\prime}, \omega\right)[28]$ :

$$
\begin{aligned}
V^{\text {ind }}(\mathbf{r}, t)= & \int \frac{d^{2} \mathbf{Q}}{(2 \pi)^{2}} \int \frac{d \omega}{2 \pi} e^{i(\mathbf{Q} \cdot \mathbf{R}-\omega t)} \\
& \times \int d z^{\prime} W^{i n d}\left(Q, z, z^{\prime}, \omega\right) \rho^{e x t}\left(Q, z^{\prime}, \omega\right),
\end{aligned}
$$

where $\rho^{\text {ext }}(Q, z, \omega)=2 \pi Z_{1} \delta(\omega-\mathbf{Q} \cdot \mathbf{v}) \delta\left(z-z_{0}\right)$ is the Fourier transform of the external charge density of Eq. (2), with respect to $\mathbf{R}$ and $t$.

Substituting Eq. (3) in Eq. (1) and making use of the parity properties of $W^{i n d}\left(Q, z, z^{\prime}, \omega\right)$, one can write the stopping power $S\left(z_{0}\right)$, defined as the energy lost per unit path length traveled by the ion at a distance $z_{0}$ from the surface, in the following way [19]:

$$
\begin{aligned}
S\left(z_{0}\right)= & \frac{1}{v} \frac{d E}{d t} \\
= & \frac{-2 Z_{1}^{2}}{v} \int \frac{d^{2} \mathbf{Q}}{(2 \pi)^{2}} \mathbf{Q} \cdot \mathbf{v} \operatorname{Im}\left\{W^{\text {ind }}\left(Q, z_{0}, z_{0}, \mathbf{Q} \cdot \mathbf{v}\right)\right\} \\
& \times \Theta(\mathbf{Q} \cdot \mathbf{v})
\end{aligned}
$$

where $\Theta(x)$ is the Heaviside step function.

$W^{\text {ind }}\left(Q, z, z^{\prime}, \omega\right)$ has a rigorous expression in terms of the exact density-density correlation function of the system $\chi\left(Q, z, z^{\prime}, \omega\right)[19]$ :

$$
\begin{aligned}
W^{\text {ind }}\left(Q, z, z^{\prime}, \omega\right)= & \left(\frac{2 \pi}{Q}\right)^{2} \int d z_{1} \int d z_{2} e^{-Q\left(\left|z_{1}-z\right|+\left|z_{2}-z^{\prime}\right|\right)} \\
& \times \chi\left(Q, z_{1}, z_{2}, \omega\right) .
\end{aligned}
$$

In general, $\chi\left(Q, z, z^{\prime}, \omega\right)$ is a very complex function, being hard to calculate exactly. Here, we use the RPA that allows us to write $\chi\left(Q, z, z^{\prime}, \omega\right)$ in terms of the density response function for noninteracting electrons, $\chi_{0}\left(Q, z, z^{\prime}, \omega\right)$ as $[24,25]$

$$
\begin{aligned}
\chi\left(Q, z, z^{\prime}, \omega\right)= & \chi_{0}\left(Q, z, z^{\prime}, \omega\right) \\
& +\int d z_{1} \int d z_{2} \chi_{0}\left(Q, z, z_{1}, \omega\right) V_{e e}\left(Q, z_{1}, z_{2}\right) \\
& \times \chi\left(Q, z_{2}, z^{\prime}, \omega\right),
\end{aligned}
$$

where the electron-electron interaction potential is $V_{e e}\left(Q, z_{1}, z_{2}\right)=(2 \pi / Q) e^{-Q\left|z_{1}-z_{2}\right|}$. Though the inclusion of local field corrections to $V_{e e}\left(Q, z_{1}, z_{2}\right)$ constitutes an improvement over the RPA response function, their effect in $S\left(z_{0}\right)$ is almost negligible [21], and therefore they are not considered here.

With $\Psi_{i} \sim e^{i \mathbf{Q}^{\prime} \cdot \mathbf{R}} \phi_{i}(z)$ and $E_{i}=\mathbf{Q}^{\prime 2} / 2+\varepsilon_{i}$ as the oneelectron wave functions and eigenvalues of the one-electron Hamiltonian, the density response function $\chi_{0}\left(Q, z, z^{\prime}, \omega\right)$ can be written in the following way $[24,25]$ :

$$
\begin{aligned}
\chi_{0}\left(Q, z, z^{\prime}, \omega\right)= & 2 \sum_{i, j} \phi_{i}(z) \phi_{j}^{*}(z) \phi_{j}\left(z^{\prime}\right) \phi_{i}^{*}\left(z^{\prime}\right) \\
& \times \int \frac{d \mathbf{Q}^{\prime}}{(2 \pi)^{2}} \frac{\Theta\left(E_{F}-E_{i}\right)-\Theta\left(E_{F}-E_{j}\right)}{E_{i}-E_{j}+(\omega+i \eta)},
\end{aligned}
$$

where $\eta$ is a positive infinitesimal, $E_{j}=\left(\mathbf{Q}^{\prime}+\mathbf{Q}\right)^{2} / 2+\varepsilon_{j}$, and $E_{F}$ is the Fermi energy of the system.

In order to analyze the effect of the above-mentioned characteristics of the $\mathrm{Cu}$ (111) surface electronic bandstructure, we compute wave functions and energies, $\phi_{i}(z)$ and $\varepsilon_{i}$, by solving the one-electron Schrödinger equation with the realistic one-dimensional model potential of Refs. $[27,29]$. This analytical potential reproduces the width and position of the energy gap at the $\bar{\Gamma}$ point $(\mathbf{Q}=0)$ and the energies of both the occupied surface state and the first image state at $\bar{\Gamma}$. Furthermore, it approaches the classical image potential far outside the surface. This potential has been used for the calculation of the lifetimes of the surface state holes $[30,31]$ and image-state electrons $[32,33]$ in the study of the resonant charge transfer in ion-surface collisions [34,35], and in the analysis of the lifetimes of excited electronic states of adsorbates at surfaces [36-38]. 


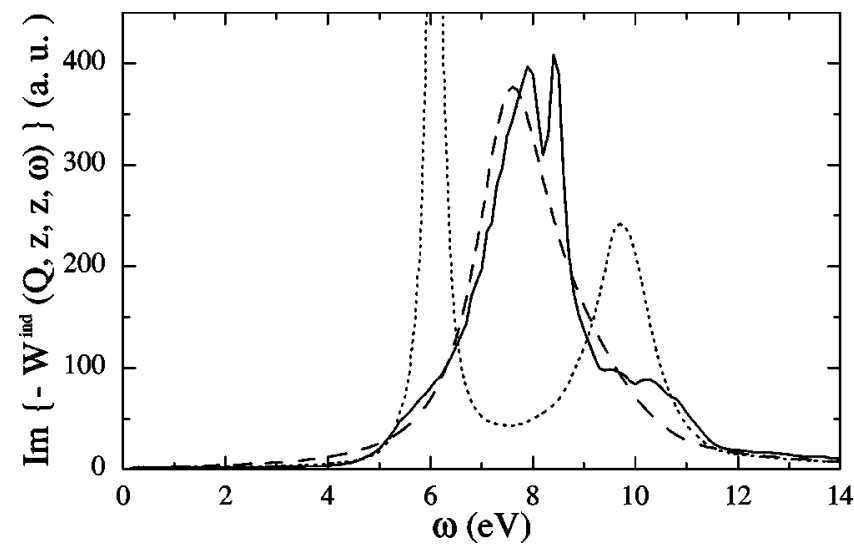

FIG. 1. $\operatorname{Im}\left\{-W^{\text {ind }}(Q, z, z, \omega)\right\}$ of the $\mathrm{Cu}(111)$ surface as a function of the energy $\omega$, for $Q=0.065$ a.u. and the distance $z=2$ a.u. measured from the topmost atomic layer. The solid line shows the results obtained when we include the surface band-structure of the target. The dotted line is obtained when the surface state is not included in the calculation of the surface band structure. The dashed line corresponds to a description of the surface with the jellium model.

This scheme allows us to determine the contribution of the surface state excitations to the energy loss of ions. With this aim we present calculations performed with and without including the surface state in the sum of Eq. (7) for the polarizability $\chi_{0}\left(Q, z, z^{\prime}, \omega\right)$. Finally, for comparison, we also present results obtained within the jellium model, i.e., neglecting all band-structure effects. In this latter model, the wave functions and energies $\left(\phi_{i}, \epsilon_{i}\right)$ entering Eq. (7) are calculated self-consistently using the Kohn-Sham equations [22]. In order to compare it with our band-structure model, $r_{s}=2.67$ a.u. has been used for the radius per electron in the conduction band.

\section{RESULTS AND DISCUSSION}

Figure 1 shows $\operatorname{Im}\left\{-W^{i n d}(Q, z, z, \omega)\right\}$ as a function of the energy $\omega$, for $Q=0.065$ a.u. and the distance $z=2$ a.u. measured from the topmost atomic layer. Interestingly, the results obtained within our complete band-based calculation that includes the surface state (solid line) and the jellium model (dashed line) are quite similar. In both cases, a pronounced peak is observed around $7-8.5 \mathrm{eV}$, which corresponds to the surface plasmon excitation. Nevertheless, some small differences are observed. In the band-based calculation, this broad peak presents a double peak structure. The reason for this is not clear at present, but we ascribe it to the more detailed description of the surface electronic structure that this model represents. Moreover, a small peak is observed around 10.5 $\mathrm{eV}$ in the band-based calculation, but not in the jellium case. This peak corresponds to the bulk plasmon that can still be excited (with a low probability) at this distance from the surface. Apart from this small differences the results are remarkably similar, considering the different description of the surface that these two models provide. More concretely, neither a projected band gap at the surface nor a surface state are present in the jellium calculation. As shown in the figure,
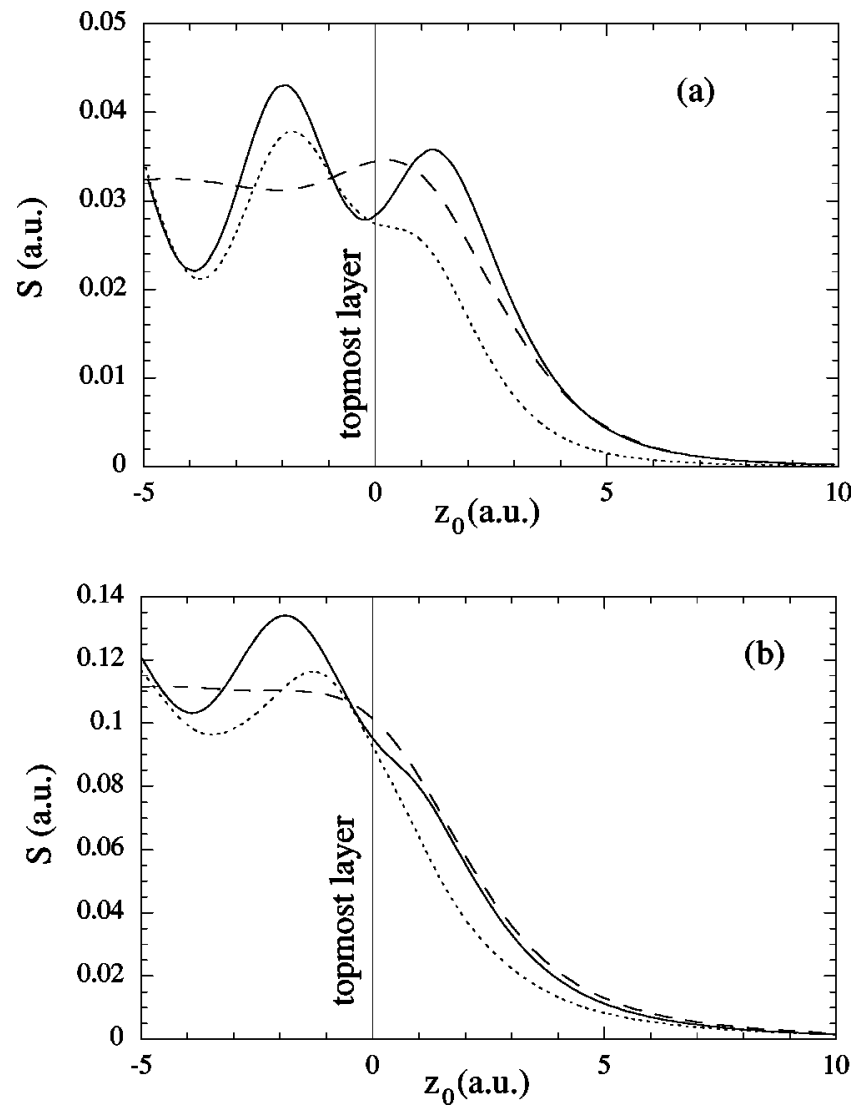

FIG. 2. Stopping power $S$ of a proton traveling parallel to the $\mathrm{Cu}$ (111) surface as a function of its distance $z_{0}$ to the topmost atomic layer. The proton velocity is (a) $v=0.25$ a.u. and (b) $v=2$ a.u.. The $\mathrm{Cu}$ (111) surface is described with different models: in solid (dotted) lines we use the surface band-structure model in which the surface state is (not) included; in dashed lines the surface is described with the jellium model (see text for details).

$\operatorname{Im}\left\{-W^{\text {ind }}(Q, z, z, \omega)\right\}$ becomes a completely different function when the surface state is not included in the band-based calculation (dotted line). In this case, two different peaks appear around $9.5 \mathrm{eV}$ and $6 \mathrm{eV}$. These peaks correspond to the bulk and surface plasmons, respectively, that are excited at lower energies than in the other calculations. This energy shift is due to the reduction of electronic density, since the surface state is not included in the calculation. Furthermore, the large strength of the bulk plasmon excitation predicted by this model for a position outside the surface is remarkable. This is an unphysical effect related to the incorrect description of the surface since we are neglecting the surface state.

In Figs. 2(a) and 2(b) we present the results obtained for the stopping power of a proton $\left(Z_{1}=1\right)$ traveling parallel to the $\mathrm{Cu}(111)$ surface as a function of the distance $z_{0}$ for two different velocities $v=0.25$ a.u. and $v=2$ a.u., respectively. Though, the linear response theory is not expected to be applicable for $v=0.25$ a.u., due to the strong perturbation that a slow ion represents, we show these results in order to obtain a physical insight on the different approximations. Negative values of $z_{0}$ correspond to positions inside the solid. In the two models based on the band-structure calculations, with (solid line) and without (dotted line) surface 
state, the stopping power shows oscillations in the inner part of the solid, reflecting the layer structure of the target. On the contrary, the jellium model (dashed line) tends to a constant value inside the solid, which is the average value of the oscillations. Outside the topmost layer, in the interesting region for specularly reflected protons, the complete bandstructure calculation and the jellium model give very similar results. The differences are quantitatively small and limited to the region close to the surface. This can be explained by the observations of Fig. 1 and by the fact that the electronic density profile at the surface in the jellium model is similar to that obtained when the surface state is included in the calculation. In this region, it is also observed that when the surface state is neglected, the stopping power is underestimated. In some respect, the presence of the surface state compensates the effect of the band gap. Remarkably, this happens not only in the high-velocity case but also at low velocities for which the details of the band structure and the subsequent low energy excitation spectra would have been expected to influence in a more significant way the values of the stopping power. In this respect, we want to emphasize that in the case of the jellium model, it is important to perform the self-consistent density functional calculation for a smooth and finite potential barrier, otherwise this model would predict unrealible values for the stopping power. For instance, Ref. [39] shows that the SRM underestimates the stopping power at large distances from the surface because the induced polarization charge is confined to the surface by the infinite potential barrier.

Figures 3(a) and 3(b) show the total energy loss of specularly reflected protons as a function of the grazing angle of incidence. The results are obtained by integrating the distance dependent stopping power along the trajectory of the projectile. Here, we consider that the projectiles are reflected due to their repulsive interaction with the average planar potential constructed in terms of the parametrized atomic Ziegler-Biersack-Littmark universal potential [40]. The image potential has not been considered in the calculation of the trajectory. To be consistent one should use different image potentials for each of the different models. This would imply slightly different proton trajectories with the same angle of incidence. Since in this work we are mostly interested in the discussion of the results of the different models for the stopping power, we compare results obtained for the same trajectory. Nevertheless, one may expect that the inclusion of the image potential would only affect considerably the results obtained for the lowest angles of incidence for which the distances of closest approach are far from the surface [12]. As could be expected from the analysis of Fig. 2, the total energy loss is almost the same in the complete bandstructure calculation and in the jellium model. Therefore, we conclude that the jellium model is a reliable approximation for the study of the energy loss of grazing incident protons in the $\mathrm{Cu}$ (111) surface in all the range of velocities. This is a remarkable fact considering the presence of the surface band gap. On the contrary, when the surface state is not included in the calculation, the total energy loss is clearly underestimated.

Finally, we would like to point out that here we have
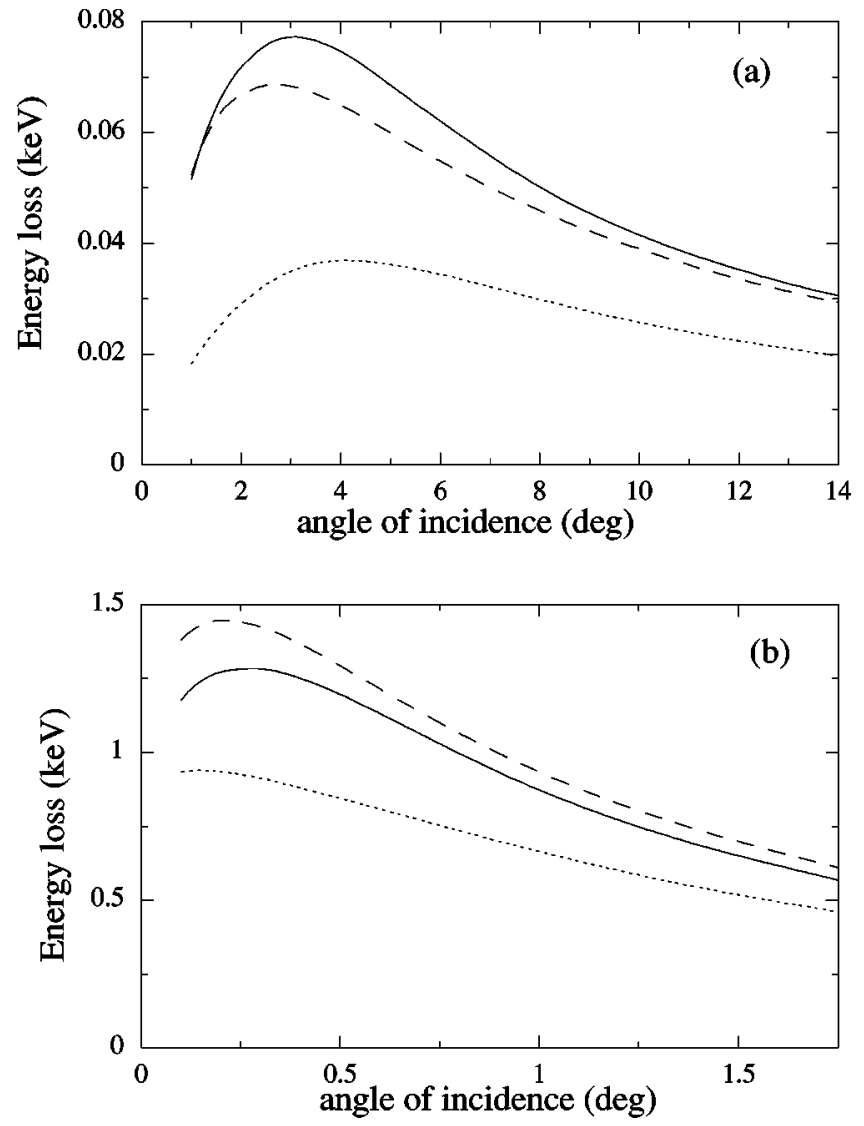

FIG. 3. Energy loss of protons specularly reflected at the $\mathrm{Cu}$ (111) surface as a function of the angle of incidence. The proton velocity is (a) $v=0.25$ a.u. and (b) $v=2$ a.u. The $\mathrm{Cu}$ (111) surface is described with different models: in solid (dotted) lines we use the surface band-structure model in which the surface state is (not) included; in dashed lines the surface is described with the jellium model (see text for details).

limited ourselves to the study of the conduction-band excitations of $\mathrm{Cu}(111)$, using different approximations. In order to compare our theoretical results with experiments, the excitation of $3 \mathrm{~d}$ electrons should also be accounted for. The $3 d$ electrons constitute an additional excitation channel for this system, which should be added to the conduction-band contribution that we have analyzed.

\section{CONCLUSION}

In summary, we have calculated the stopping power and energy loss of protons scattered by the $\mathrm{Cu}$ (111) surface, using information about the band structure of the solid. In particular, the relevance of the surface state has clearly been shown: when it is neglected the energy loss is largely underestimated. However, when it is included in the calculation, the results obtained are similar to those obtained within the jellium model. This leads us to conclude that concerning the energy loss of protons scattered off the $\mathrm{Cu}$ (111) surface (1) the surface state compensates the presence of the projected surface band gap and (2) the jellium model represents a good approximation. These conclusions are expected to be valid for other metallic surfaces with surface states. 


\section{ACKNOWLEDGMENTS}

The authors thank Professor P. M. Echenique for many stimulating discussions. We acknowledge partial support by the Eusko Jaurlaritza, Iberdrola S. A., Gipuzkoako Foru Aldundia, and Euskal Herriko Unibertsitatea. M.A. and J.I.J. also acknowledge support by the Spanish DGICYT (Project No. BFM-2001-0076).
[1] H. Winter, Phys. Rep. 367, 387 (2002).

[2] K. Kimura, M. Hasegawa, and M. Mannami, Phys. Rev. B 36, 7 (1987).

[3] H. Winter, M. Wilke, and M. Bergomaz, Nucl. Instrum. Methods Phys. Res. B 125, 124 (1997).

[4] H. Winter, M. Wilke, and R. Zimny, Nucl. Instrum. Methods Phys. Res. B 100, 396 (1995).

[5] J.I. Juaristi, A. Arnau, P.M. Echenique, C. Auth, and H. Winter, Phys. Rev. Lett. 82, 1048 (1999).

[6] J.I. Juaristi, A. Arnau, P.M. Echenique, C. Auth, and H. Winter, Nucl. Instrum. Methods Phys. Res. B 157, 87 (1999).

[7] A. Robin, W. Heiland, J. Jensen, J.I. Juaristi, and A. Arnau, Phys. Rev. A 64, 052901 (2001).

[8] P.M. Echenique and J.B. Pendry, J. Phys. C 8, 2936 (1975).

[9] R. Nuñez, P.M. Echenique, and R.H. Ritchie, J. Phys. C 13, 429 (1980)

[10] N. Zabala and P.M. Echenique, Ultramicroscopy 32, 327 (1990).

[11] J.I. Juaristi and F.J. García de Abajo, Nucl. Instrum. Methods Phys. Res. B 90, 252 (1994).

[12] J.I. Juaristi, F.J. García de Abajo, and P.M. Echenique, Phys. Rev. B 53, 13839 (1996).

[13] Y.-H. Song and Y.-N. Wang, Nucl. Instrum. Methods Phys. Res. B 153, 186 (1999).

[14] Y.-H. Song, Y.-N. Wang, and Z.L. Miŝsković, Phys. Rev. A 63, 052902 (2001).

[15] R.H. Ritchie and A.L. Marusak, Surf. Sci. 4, 234 (1966).

[16] D. Wagner, Z. Naturforsch. A 21A, 634 (1966).

[17] M.S. Gravielle, Phys. Rev. A 58, 4622 (1998).

[18] M.S. Gravielle and J.E. Miraglia, Phys. Rev. A 65, 022901 (2002).

[19] M.A. Cazalilla and F.J. García de Abajo, Nucl. Instrum. Methods Phys. Res. B 125, 106 (1997).

[20] M.A. Cazalilla and J.I. Juaristi, Nucl. Instrum. Methods Phys. Res. B 157, 104 (1999).
[21] A. García-Lekue and J.M. Pitarke, Phys. Rev. B 64, 035423 (2001).

[22] W. Kohn and L.J. Sham, Phys. Rev. 140, A1133 (1965).

[23] P. Hohenberg and W. Kohn, Phys. Rev. 136, B864 (1964).

[24] A.G. Eguiluz, Phys. Rev. Lett. 51, 1907 (1983).

[25] A.G. Eguiluz, Phys. Rev. B 31, 3303 (1985).

[26] A. Zangwill and P. Soven, Phys. Rev. A 21, 1561 (1980).

[27] E.V. Chulkov, V.M. Silkin and P.M. Echenique, Surf. Sci. 437, 330 (1999).

[28] P.M. Echenique, V.H. Ponce, M.E. Uranga, and F.J. García de Abajo, Nucl. Instrum. Methods Phys. Res. B 96, 583 (1995).

[29] E.V. Chulkov, V.M. Silkin, and P.M. Echenique, Surf. Sci. 391, L1217 (1997).

[30] J. Kliewer, R. Berndt, E.V. Chulkov, V.M. Silkin, P.M. Echenique, and S. Crampin, Science 288, 1399 (2000).

[31] P.M. Echenique, J. Osma, V.M. Silkin, E.V. Chulkov, and J.M. Pitarke, Appl. Phys. A: Mater. Sci. Process. 71, 503 (2000).

[32] E.V. Chulkov, I. Sarría, V.M. Silkin, J.M. Pitarke, and P.M. Echenique, Phys. Rev. Lett. 80, 4947 (1998).

[33] P.M. Echenique, J.M. Pitarke, E.V. Chulkov, and A. Rubio, Chem. Phys. 251, 1 (2000).

[34] A.K. Kazansky, A.G. Borisov, and J.P. Gauyacq, Nucl. Instrum. Methods Phys. Res. B 157, 21 (1999).

[35] T. Hecht, H. Winter, A.G. Borisov, J.P. Gauyacq, and A.K. Kazansky, Phys. Rev. Lett. 84, 2517 (2000).

[36] J.P. Gauyacq, A.G. Borisov, and G. Raseev, Surf. Sci. 490, 99 (2001).

[37] A.G. Borisov, A.K. Kazansky, and J.P. Gauyacq, Phys. Rev. B 64, 201105 (2001).

[38] A.G. Borisov, J.P. Gauyacq, E.V. Chulkov, V.M. Silkin, and P.M. Echenique, Phys. Rev. B 65, 235434 (2002).

[39] A. Alducin, V.M. Silkin, J.I. Juaristi, and E.V. Chulkov, Nucl. Instrum. Methods Phys. Res. B 193, 585 (2002).

[40] J.F. Ziegler, J.P. Biersack, and U. Littmark, The Stopping and Range of Ions in Solids (Pergamon Press, New York, 1985). 